\title{
Spacecraft Pose Estimation Based on Different Camera Model
}

\author{
Li-Dong Mo ( $\nabla$ molidong@126.com ) \\ Harbin Institute of Technology \\ Guan-Xin Chi \\ Harbin Institute of Technology \\ Zhen-Qing Zhao \\ Northeast Agriculture University
}

\section{Original Article}

Keywords: Spacecraft pose estimation, Weak perspective projection, Optimal solution

Posted Date: August 21st, 2020

DOI: https://doi.org/10.21203/rs.3.rs-62297/v1

License: (c) (1) This work is licensed under a Creative Commons Attribution 4.0 International License. Read Full License 


\section{Title page}

\section{Spacecraft Pose Estimation Based on Different Camera Model}

Li-Dong Mo, is currently a $\mathrm{PhD}$ candidate at School of mechanical and electrical engineering, Harbin Institute of Technology, Heilongjiang, China.

Tel: +86-182-22231667; E-mail: molidong@126.com

Guan-Xin Chi, is currently a professor at School of mechanical and electrical engineering, Harbin Institute of Technology, Heilongjiang, China. He received his PhD degree in Harbin Institute of Technology, China, in 1999.

Tel: +86-139-36130623; E-mail: chigx@hit.edu.cn

Zhen-Qing Zhao, is currently a teacher at Northeast Agricultural University, Heilongjiang, China.

Tel: +86-186-46031686; E-mail: zzq725@126.com

Corresponding author: Li-Dong Mo E-mail: molidong@126.com 


\title{
ORIGINAL ARTICLE
}

\section{Spacecraft Pose Estimation Based on Different Camera Model}

\author{
Li-Dong Mo ${ }^{1 *} \cdot$ Guan-Xin $\mathrm{Chi}^{2} \cdot$ Zhen-Qing Zhao ${ }^{3}$
}

Received June xx, 201x; revised February xx, 201x; accepted March xx, 201x

(C) Chinese Mechanical Engineering Society and Springer-Verlag Berlin Heidelberg 2017

\begin{abstract}
Spacecraft pose estimation is an important technology for spacecraft to maintain or change its orientation in space. For spacecraft pose estimation, when two spacecraft are relatively far away, the depth information of the space point is less than that of measuring distance, so that the camera model can be seen as a weak perspective projection model. In this paper, a spacecraft pose estimation algorithm based on four symmetrical points of the spacecraft outline is proposed. Analytical solution of spacecraft pose is obtained by solving the weak perspective projection model, which can meet the requirements of the measurement model when there is a long measurement distance. Optimal solution is obtained from the weak perspective projection model to the perspective projection model, which can meet the measurement requirement when the measuring distance is close. The simulation results show that the proposed algorithm can get better results even though the noise is large.
\end{abstract}

Keywords: Spacecraft pose estimation - Weak perspective projection - Optimal solution

\section{Introduction}

With the development of space technology, more and more space missions involve the relative position measurement of two spacecraft [1-3], such as the space assembly, space satellite repair, fuel injection, satellite capturing and tracking, space interception and so on. The measurement of spacecraft relative position plays a very important role in maintaining or changing the spacecraft's orientation in space so that to complete the space mission.

For relative position measurement, vision has some advantages in weight, volume, power consumption and cost

Li-Dong Mo

molidong@126.com

1 School of mechanical and electrical engineering, Harbin Institute of Technology, Heilongjiang, 150001, China of equipment[4-6]. In the smart-OLEV [7-8] mission, the SMART-1 platform uses stereo cameras and lighting equipment to provide better measurement data within 5 meters, but only provides pointing data at a long distance. The Argon vision system is divided into long-distance and short-range vision sensors [9-10], which select different field of view sensors for different distances. The natural image feature recognition system developed by Johnson Space Center USES the 3D model of the target under test to calculate the relative pose [11-12], and the measurement accuracy is proportional to the relative distance. The measurement system requires to measure the relative pose information of two spacecraft at different distances for control system or other systems, and the relative distance of spacecraft varies greatly, reaching more than 20 times. So, the spacecraft pose estimation has the following characteristics:

1. When the two spacecraft are relatively far away, the depth information of feature points on the target spacecraft and the distance between the feature points are less than the relative distance between the two spacecraft.

2. Since the focal length of the camera is fixed, the accuracy of feature points extraction is getting lower with the increase of the relative distance between the two spacecraft.

Based on the above reasons, when the two spacecraft are far apart, the pose measurement accuracy will be reduced. At present, there are two main algorithms for pose estimation:

1) Based on cooperative space measurement. First, Analytical algorithm based on the perspective projection camera model, such as perspective-n-point (PnP) [13-15],

2 School of mechanical and electrical engineering, Harbin Institute of Technology, Heilongjiang, 150001, China

Northeast Agricultural University, Heilongjiang, 150001, China 
direct linear transformation (DLT) [16,17]. Through these algorithms, the pose of spacecraft can be solved directly. However, the accuracy of spacecraft pose obtained by analytical algorithm is unsatisfactory. and optimization algorithm based on camera nonlinear model, such as GaussNewton algorithm, Levenberg-Marqurdt algorithm and orthogonal iteration (OI) algorithm [18-19]. These algorithms need a good initial solution to optimize. Therefore, we hope to obtain high-precision analytical solutions through analytical algorithms. 2) Based on noncooperative space measurement, the transformation of pose is calculated by using pattern matching and $3 \mathrm{D}$ point cloud technology[20-23].

In this paper, a spacecraft pose estimation algorithm based on target geometric constraints of the spacecraft outline is proposed. In order to reduce the influence of distance on measurement accuracy, this paper simplifies the camera measurement model. The simulation results show that the proposed algorithm has the effect of image feature error of 0.1 pixel to 1 pixel from 1 meter to 20 meters.

\section{POSE ESTIMATION ALGORITHM}

Spacecraft pose estimation is based on the relationship between the target spacecraft points and the corresponding image points, and calculates the relative pose of the target spacecraft coordinate system and camera coordinate system by using the multi-point correspondence relationship.

The mapping relations between the target spacecraft point and image point can be described by two mathematical mappings: 1) rigid transformation. The space points in the space coordinate system and camera coordinate system follow rigid body transformation, namely rotation transformation and translation transformation. Because the camera is installed on the tracker spacecraft, the relative pose relationship between the tracker spacecraft and the target spacecraft can be described by the pose relationship between the target spacecraft and the camera coordinate system. 2) Camera model, the relationship between 3D space points in the camera coordinates and the projection $2 \mathrm{D}$ image points on the camera image plane.

\subsection{Algorithm model}

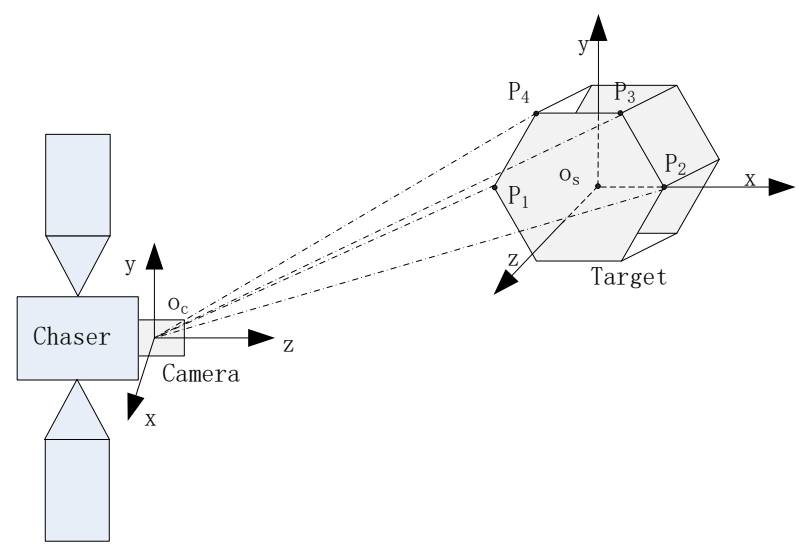

Figure 1 Target spacecraft and camera coordinate system

In order to construct the spacecraft pose estimation algorithm model, four coplanar symmetry points are used to calculate the spacecraft pose. The target spacecraft coordinate system is Os-xyz. There are four points for $P_{i}^{s}$ $i=1, \cdots, 4$. Four points in target spacecraft coordinates are

$$
P_{1}^{s}=\left[\begin{array}{l}
a \\
0 \\
d
\end{array}\right] \quad P_{2}^{s}=\left[\begin{array}{c}
-a \\
0 \\
d
\end{array}\right] \quad P_{3}^{s}=\left[\begin{array}{l}
c \\
b \\
d
\end{array}\right] \quad P_{4}^{s}=\left[\begin{array}{c}
-c \\
b \\
d
\end{array}\right],
$$

where $\mathrm{a}, \mathrm{b}, \mathrm{c}, \mathrm{d}$ are known values, The relationship between $P_{\mathrm{i}}^{s} \quad$ and the points in camera coordinate system $P_{\mathrm{i}}^{c}$ are given by

$$
P_{i}^{c}=R P_{i}^{s}+T
$$

where

$$
R_{3 \times 3}=\left[\begin{array}{c}
I \\
J \\
K
\end{array}\right]=\left[\begin{array}{lll}
r_{11} & r_{12} & r_{13} \\
r_{21} & r_{22} & r_{23} \\
r_{31} & r_{32} & r_{33}
\end{array}\right] \quad T_{3 \times 1}=\left[\begin{array}{c}
t_{x} \\
t_{y} \\
t_{z}
\end{array}\right],
$$

\subsection{Camera model}

The fixed focus lens camera model can be simplified to a single lens model. According to the principle of optical, space points $P_{i}^{c}\left(x_{i}^{c}, y_{i}^{c}, z_{i}^{c}\right)$, image points $p_{i}\left(u_{i}, v_{i}\right)$ and the camera origin $O_{\mathrm{c}}$ are located on the same line. Therefore camera model is called a pinhole camera model, also known 
as the perspective projection model.

$$
u_{i}=\frac{f x_{i}^{c}}{z_{i}^{c}} \quad v_{i}=\frac{f x_{i}^{c}}{z_{i}^{c}}
$$

where $f$ is the camera focal distance. The spacecraft pose estimation model is

$$
\left[\begin{array}{c}
u_{i} z_{i}^{c} / f \\
v_{i} z_{i}^{c} / f \\
z_{i}^{c}
\end{array}\right]=\left[\begin{array}{c}
I P_{i}^{s} \\
J P_{i}^{s} \\
K P_{i}^{s}
\end{array}\right]+\left[\begin{array}{c}
t_{x} \\
t_{y} \\
t_{z}
\end{array}\right],
$$

Form equation (2), we get the relationship between $z_{i}^{c}$ and $t_{z}$

$$
z_{i}^{c}=t_{z}(1+\varepsilon) \quad \varepsilon=K P^{s} / t_{z},
$$

Finally, we have

$$
\begin{aligned}
& {\left[\begin{array}{cccc}
\frac{u_{1}}{f} t_{z}\left(1+\varepsilon_{1}\right) & \frac{u_{2}}{f} t_{z}\left(1+\varepsilon_{2}\right) & \frac{u_{3}}{f} t_{z}\left(1+\varepsilon_{3}\right) & \frac{u_{4}}{f} t_{z}\left(1+\varepsilon_{4}\right) \\
\frac{v_{1}}{f} t_{z}\left(1+\varepsilon_{1}\right) & \frac{v_{2}}{f} t_{z}\left(1+\varepsilon_{2}\right) & \frac{v_{3}}{f} t_{z}\left(1+\varepsilon_{3}\right) & \frac{v_{4}}{f} t_{z}\left(1+\varepsilon_{4}\right) \\
t_{z}\left(1+\varepsilon_{1}\right) & t_{z}\left(1+\varepsilon_{2}\right) & t_{z}\left(1+\varepsilon_{3}\right) & t_{z}\left(1+\varepsilon_{4}\right)
\end{array}\right]} \\
& =\left[\begin{array}{llll}
-a r_{11}+d r_{13} & a r_{11}+d r_{13} & c r_{11}+b r_{12}+d r_{13} & -c r_{11}+b r_{12}+d r_{13} \\
-a r_{21}+d r_{23} & a r_{21}+d r_{23} & c r_{21}+b r_{22}+d r_{23} & -c r_{21}+b r_{22}+d r_{23} \\
-a r_{31}+d r_{33} & a r_{31}+d r_{33} & c r_{31}+b r_{33}+d r_{33} & -c r_{31}+b r_{33}+d r_{33}
\end{array}\right], \\
& +\left[\begin{array}{l}
t_{x} \\
t_{y} \\
t_{z}
\end{array}\right]
\end{aligned}
$$

According to the symmetry properties of points, we have

$$
\left\{\begin{array} { l } 
{ r _ { 1 1 } = k _ { 1 } t _ { z } } \\
{ r _ { 2 1 } = k _ { 2 } t _ { z } }
\end{array} \quad \left\{\begin{array} { l } 
{ r _ { 1 2 } = k _ { 3 } t _ { z } } \\
{ r _ { 2 2 } = k _ { 4 } t _ { z } }
\end{array} \quad \left\{\begin{array}{l}
r_{13}=k_{5} t_{z}-t_{x} / d \\
r_{23}=k_{6} t_{z}-t_{y} / d
\end{array},\right.\right.\right.
$$

where

$$
\left\{\begin{array}{l}
k_{1}=\frac{\left(u_{2}\left(1+\varepsilon_{2}\right)-u_{1}\left(1+\varepsilon_{1}\right)\right)}{2 a f} \\
k_{2}=\frac{\left(v_{2}\left(1+\varepsilon_{2}\right)-v_{1}\left(1+\varepsilon_{1}\right)\right)}{2 a f} \\
k_{3}=\frac{\left(u_{3}\left(1+\varepsilon_{3}\right)+u_{4}\left(1+\varepsilon_{4}\right)-u_{2}\left(1+\varepsilon_{2}\right)-u_{1}\left(1+\varepsilon_{1}\right)\right)}{2 b f} \\
k_{4}=\frac{\left(v_{3}\left(1+\varepsilon_{3}\right)+v_{4}\left(1+\varepsilon_{4}\right)-v_{2}\left(1+\varepsilon_{2}\right)-v_{1}\left(1+\varepsilon_{1}\right)\right)}{2 b f} \\
k_{5}=\frac{\left(u_{2}\left(1+\varepsilon_{2}\right)+u_{1}\left(1+\varepsilon_{1}\right)\right)}{2 d f} \\
k_{6}=\frac{\left(v_{2}\left(1+\varepsilon_{2}\right)-v_{1}\left(1+\varepsilon_{1}\right)\right)}{2 d f}
\end{array}\right.
$$

\subsection{Simplified model}

When the two spacecraft are relatively far away, the accuracy of image feature extraction is low, and the depth information of feature points to the target spacecraft can be ignored. Camera model can be approximated by a simplified perspective projection model [24,25]. Then, we have

$$
z_{i}^{c}=t_{z} \quad i=1,2,3,4
$$

Simplified perspective projection refers to the projection on the plane parallel to the imaging plane through the origin of the target spacecraft. Therefore, it ignores the depth of the target spacecraft point relative to the origin of the target spacecraft. When the two spacecraft are relatively far away, the neglect error is very small. From equations (8), we have

$$
\begin{aligned}
& k_{1}=\frac{\left(u_{2}-u_{1}\right)}{2 a f} k_{2}=\frac{\left(v_{2}-v_{1}\right)}{2 a f} k_{3}=\frac{\left(u_{3}+u_{4}-u_{2}-u_{1}\right)}{2 b f} \\
& k_{4}=\frac{\left(v_{3}+v_{4}-v_{2}-v_{1}\right)}{2 b f} k_{5}=\frac{\left(u_{2}+u_{1}\right)}{2 d f} k_{6}=\frac{\left(v_{2}-v_{1}\right)}{2 d f}
\end{aligned}
$$

$k_{i}$ value can be calculated by image points.

Equations (7) contains 9 variables and 6 equations, it cannot be solved directly. The rotation matrix $R$ have the following constraints 


$$
\left\{\begin{array}{l}
r_{11}{ }^{2}+r_{12}{ }^{2}+r_{13}{ }^{2}=1 \\
r_{21}{ }^{2}+r_{22}{ }^{2}+r_{23}{ }^{2}=1 \\
r_{31}{ }^{2}+r_{32}{ }^{2}+r_{33}{ }^{2}=1 \\
r_{11} r_{21}+r_{12} r_{22}+r_{13} r_{23}=0 \\
r_{31} r_{21}+r_{32} r_{22}+r_{33} r_{23}=0 \\
r_{11} r_{31}+r_{12} r_{32}+r_{13} r_{33}=0
\end{array},\right.
$$

From equation (12-6), (12-1), and (12-3), we have

$$
\left(r_{11} r_{21}-r_{12} r_{22}\right)^{2}-\left(r_{11}^{2}+r_{12}{ }^{2}+r_{21}{ }^{2}+r_{22}{ }^{2}\right)+1=0,
$$

From equations (8) and (13), we have

$$
\left(k_{1} k_{3}-k_{2} k_{4}\right)^{2} t_{z}^{4}-\left(k_{1}^{2}+k_{2}^{2}+k_{3}^{2}+k_{4}^{2}\right) t_{z}^{2}+1=0,
$$

Equation (14) is a quartic equation. There are four roots, remove two negative roots, according to the relationship between roots and coefficients, two positive roots meet:

$$
\begin{aligned}
& \text { Condition 1: } t_{z 1}^{2} \leq \frac{k_{1}^{2}+k_{3}^{2}}{\left(k_{1} k_{3}-k_{2} k_{4}\right)^{2}}, \\
& \text { Condition 2: } t_{z 2}^{2} \geq \frac{k_{2}^{2}+k_{4}^{2}}{\left(k_{1} k_{3}-k_{2} k_{4}\right)^{2}},
\end{aligned}
$$

Only when the rotation angle is greater than $60^{\circ}$ can condition 2 be satisfied, so the result of condition 1 is selected.

Rotation matrix $\mathrm{R}$ can be described by four quaternion parameters $\left(q_{0}, q_{1}, q_{2}, q_{3}\right)$,

$$
R=\left[\begin{array}{ccc}
q_{0}^{2}+q_{1}^{2}-q_{2}^{2}-q_{3}^{2} & 2\left(q_{1} q_{2}+q_{3} q_{0}\right) & 2\left(q_{1} q_{3}-q_{2} q_{0}\right) \\
2\left(q_{1} q_{2}-q_{3} q_{0}\right) & q_{0}^{2}-q_{1}^{2}+q_{2}^{2}-q_{3}^{2} & 2\left(q_{2} q_{3}+q_{1} q_{0}\right) \\
2\left(q_{1} q_{3}+q_{2} q_{0}\right) & 2\left(q_{2} q_{3}-q_{1} q_{0}\right) & q_{0}^{2}-q_{1}^{2}-q_{2}^{2}+q_{3}^{2}
\end{array}\right]
$$

Assumed that,

$$
\begin{aligned}
& \beta=\frac{1}{2}\left(r_{32}-\left(r_{12} r_{31}-r_{11} r_{32}\right)\right) \\
& =\frac{1}{2}\left(k_{4} t_{y}-k_{3} k_{2} t_{z}^{2}-k_{1} k_{4} t_{z}^{2}\right)=2 q_{1} q_{2},
\end{aligned}
$$

Form equations (14), we have

$$
\left\{\begin{array}{l}
q_{1}^{2}+q_{2}^{2}=\frac{1}{2}\left(1+r_{11}\right) \\
q_{1}^{2}-q_{2}^{2}=-\frac{1}{2} \sqrt{\left(1+r_{11}\right)^{2}-4 \beta^{2}} \\
q_{1} r_{12}+q_{2} r_{31}=2 q_{4}\left(q_{2}^{2}-q_{1}^{2}\right) \\
q_{2} r_{12}+q_{1} r_{31}=2 q_{3}\left(q_{2}^{2}-q_{1}^{2}\right)
\end{array}\right.
$$

Then, we have

$$
\begin{aligned}
& \left\{\begin{array}{l}
q_{1}=\frac{1}{2} \sqrt{1+k_{1} t_{z}-\sqrt{\left(1+k_{1} t_{z}\right)^{2}-4 \beta^{2}}} \\
q_{2}=\frac{\beta}{2 q_{1}} \\
q_{3}=\frac{q_{2} k_{3}+q_{1} k_{2}}{2\left(q_{2}^{2}-q_{1}^{2}\right)} t_{z} \\
q_{4}=\frac{q_{1} k_{3}+q_{2} k_{2}}{2\left(q_{2}^{2}-q_{1}^{2}\right)} \\
t_{x}=\frac{t_{z}}{2 f}\left(u_{1}\left(1+\varepsilon_{1}\right)+u_{2}\left(1+\varepsilon_{2}\right)\right)-d r_{13} \\
t_{y}=\frac{t_{z}}{2 f}\left(v_{1}\left(1+\varepsilon_{1}\right)+v_{2}\left(1+\varepsilon_{2}\right)\right)-d r_{33} \\
t_{x}=\frac{t_{z}}{2 f}\left(u_{1}\left(1+\varepsilon_{1}\right)+u_{2}\left(1+\varepsilon_{2}\right)\right)-d r_{13} \\
t_{y}=\frac{t_{z}}{2 f}\left(v_{1}\left(1+\varepsilon_{1}\right)+v_{2}\left(1+\varepsilon_{2}\right)\right)-d r_{33}
\end{array},\right.
\end{aligned}
$$

\subsection{Optimization algorithm}

The Spacecraft pose estimation accuracy based on simplified perspective projection is poor. So, an iterative optimal algorithm to improve the solution accuracy is constructed. The optimal algorithm to improve the spacecraft pose estimation accuracy is shown in Fig. 2. 


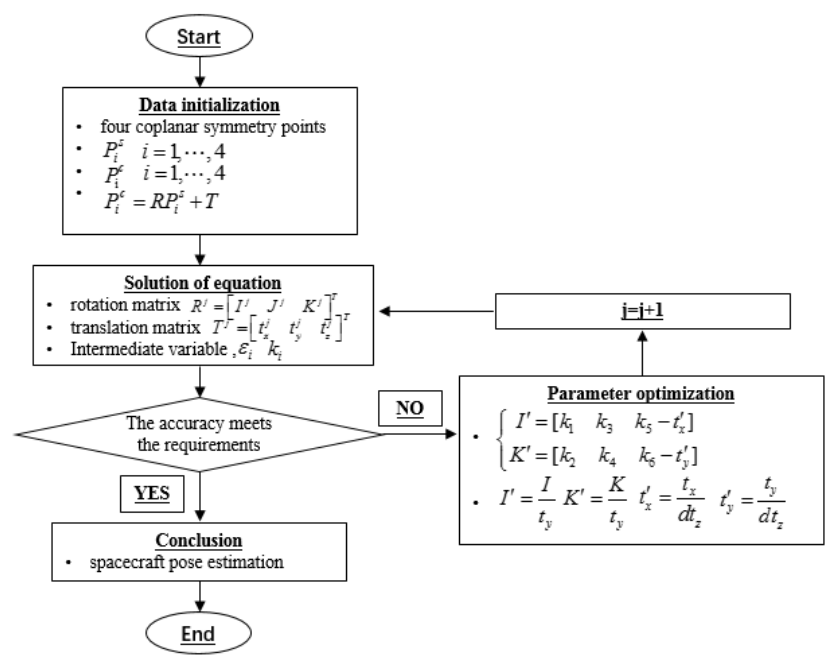

Figure 2 The optimal algorithm to improve the spacecraft pose estimation accuracy

In the algorithm , $\quad R^{j}=\left[\begin{array}{lll}I^{j} & J^{j} & K^{j}\end{array}\right]^{T}$ and $T^{j}=\left[\begin{array}{lll}t_{x}^{j} & t_{y}^{j} & t_{z}^{j}\end{array}\right]^{T}$,

$$
\begin{aligned}
& I^{j}=\frac{I^{\prime}}{\left\|I^{\prime}\right\|} \quad K^{j}=\frac{K^{\prime}}{\left\|K^{\prime}\right\|} \quad J^{j}=I^{j} \times K^{j} \\
& t_{z}^{j}=\frac{1}{2}\left(\frac{1}{\left\|I^{\prime}\right\|}+\frac{1}{\left\|K^{\prime}\right\|}\right) \quad t_{x}^{j}=d t_{z}^{j} t_{x}^{\prime} \quad t_{y}^{j}=t_{z}^{j} t_{y}^{\prime} d
\end{aligned}
$$

\section{Experimental Section}

The simulation experiment parameters are set as follows. The focal distance of camera is $12 \mathrm{~mm}$. The pixel size is $7.4 \mu \mathrm{m} \times 7.4 \mu \mathrm{m}$. The rotation matrix and translation vector are $[\varphi, \theta, \psi]=[30,30,30]\left(^{\circ}\right)$ and $T=\left[0.5 t_{z}, 0.5 t_{z}, t_{z}\right](\mathrm{m})$, where $t_{z}=1 \sim 20$. Four points in target spacecraft coordinates are

$$
P_{1}^{s}=\left[\begin{array}{c}
75 \\
0 \\
75
\end{array}\right] \quad P_{2}^{s}=\left[\begin{array}{c}
-75 \\
0 \\
75
\end{array}\right] \quad P_{3}^{s}=\left[\begin{array}{l}
30 \\
40 \\
75
\end{array}\right] \quad P_{4}^{s}=\left[\begin{array}{c}
-30 \\
40 \\
75
\end{array}\right]
$$

Simulation experiments verify the proposed algorithm from the following three aspects: 1) Analyze optimization algorithm. The iterative optimization simulation in $\mathrm{m}$ without noise. 2) Analyze the relationship between estimation accuracy and distance. Gaussian noise with mean value 0 and standard deviation 0.1 pixel is added to the projected image points to the projected image points. 3) Analyze the relationship between estimation accuracy and distance. Gaussian noise with mean value 0 and standard deviation 1 pixel is added to the projected image points to the projected image points.

The simulation results are given in Figure 3 and Figure 4. Spacecraft pose estimation error is large based on simplified perspective projection, optimization algorithm based on camera model effectively reduces the measurement error. After 10 iterations, the errors of attitude are less than $0.42^{\circ}$, the errors of position are less than $4 \mathrm{~mm}$.

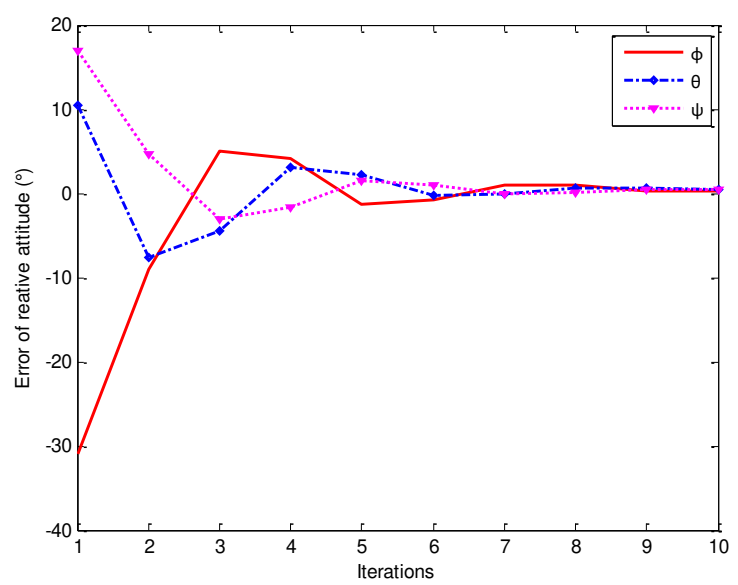

Figure.3 Attitude accuracy analysis using optimization.

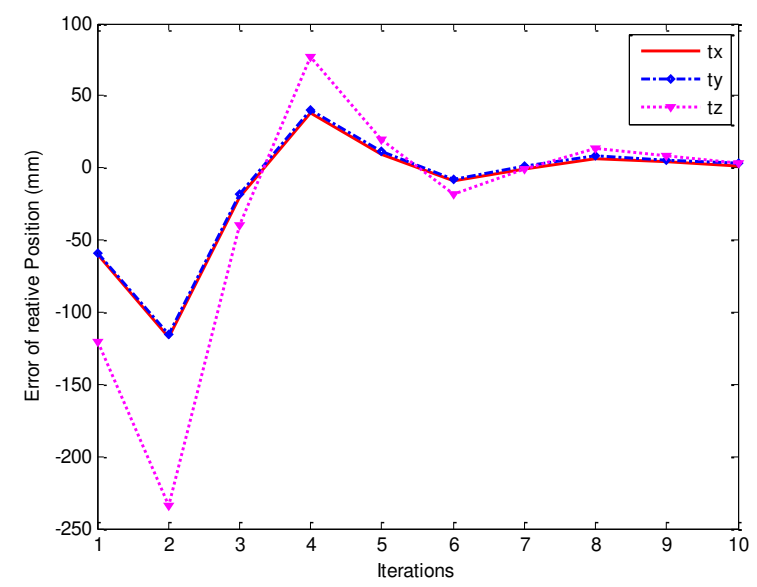

Figure.4 Position accuracy analysis using optimization.

Fig.5 and Fig.6 show estimation accuracy with mean value 0 and standard deviation 0.1 pixel noise. When $t_{z}$ is 10 meter, the error of attitude are less than $0.36^{\circ}$, the error of position is less than $19.5 \mathrm{~mm}$. When $t_{z}$ is 20 meter, the error of attitude are less than $0.65^{\circ}$, and the error of position is less than $117 \mathrm{~mm}$. The maximum pose error occurs when $t_{z}=1$ $\mathrm{m}$, the main reason is that the initial relative position accuracy is low based on simplified perspective projection model. 


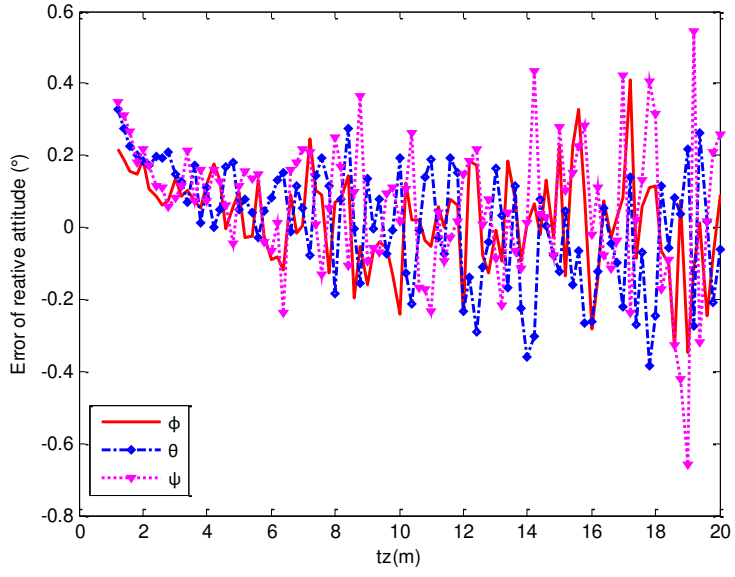

Figure.5 Attitude accuracy analysis with 0 mean and 0.1 pixel standard deviation noise

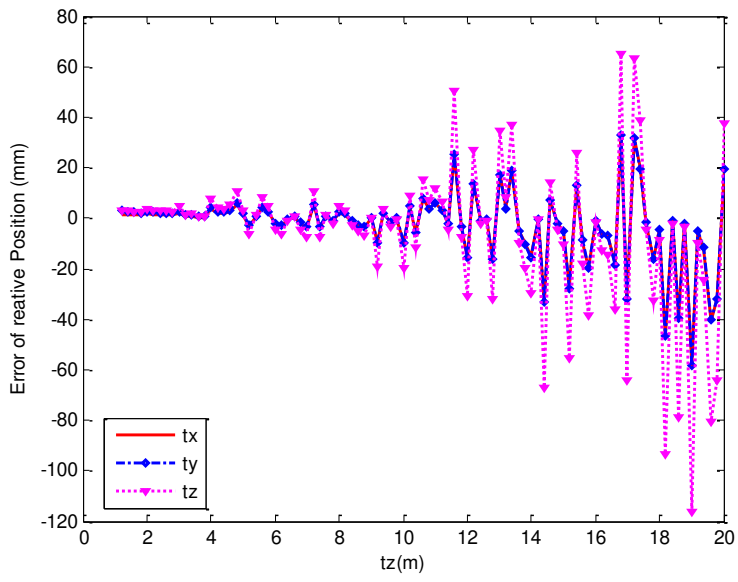

Figure.6 Position accuracy analysis with 0 mean and 0.1 pixel standard deviation noise

Fig. 7 and Fig. 8 showestimation accuracy with mean value 0 and standard deviation 1 pixel noise. When $t_{z}$ is 10 meter, the errors of attitude are less than $3^{\circ}$, the errors of position are less than $0.35 \mathrm{~m}$. When $t_{z}$ is 20 meter, the errors of attitude are less than $7.5^{\circ}$, the errors of position are less than $1 \mathrm{~m}$.

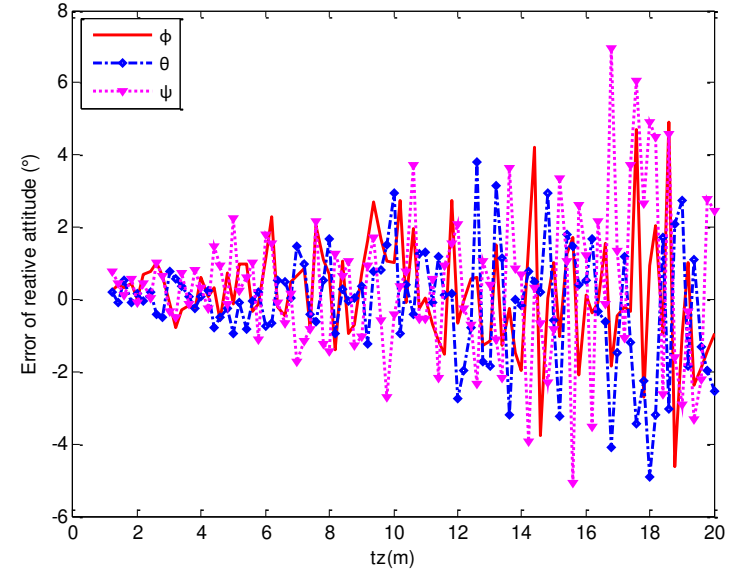

Figure.7 Attitude accuracy analysis with 0 mean and 1 pixel standard deviation noise

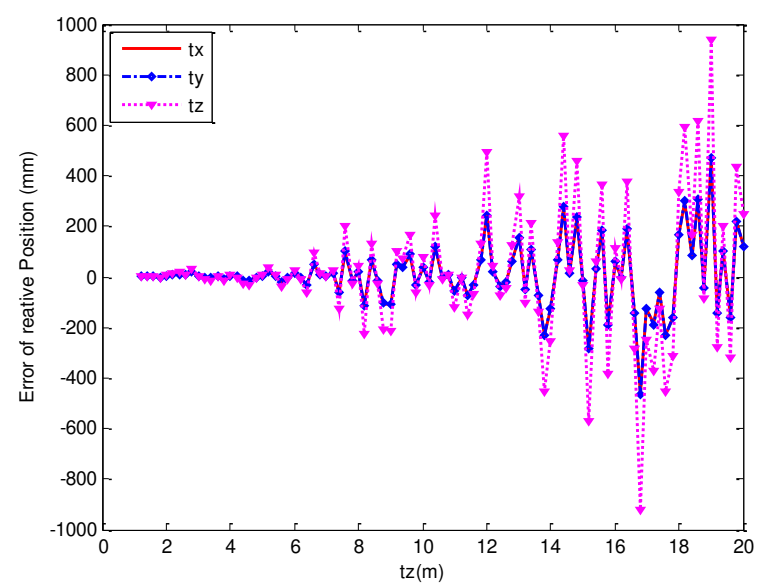

Figure.8 Position accuracy analysis with 0 mean and 1 pixel standard deviation noise

\section{Conclusions}

In order to meet the requirements of pose estimation accuracy for the spacecraft relative distance change from far to near, we propose a model based on two different camera models. In this algorithm, the initial value of spacecraft pose is calculated by the simplified perspective projection model, and the results are further optimized by the perspective projection model. The simulation results show the errors of pose estimation are less than $0.8^{\circ}, 117 \mathrm{~mm}$ when image points have 0 mean and 0.1 pixel standard deviation, the errors of pose estimation are less than $7.5^{\circ}, 1 \mathrm{~m}$ when image points have 0 mean and 1 pixel standard deviation. Estimation accuracy can meet the demand of spacecraft missions. 


\section{Declaration}

\section{Acknowledgements}

The authors sincerely thanks to Professor Chi of Harbin Institute of Technology for his critical discussion and reading during manuscript preparation.

\section{Funding}

Not applicable

\section{Availability of data and materials}

The datasets supporting the conclusions of this article are included within the article.

\section{Authors' contributions}

The author' contributions are as follows: Li-Dong Mo was in charge of the whole algorithm and wrote the manuscript; Guan-Xin Chi provided suggestions and rectification ideas; Zhen-Qing Zhao assisted with sampling and laboratory analyses.

\section{Competing interests}

The authors declare no competing financial interests.

\section{Consent for publication}

Not applicable

\section{Ethics approval and consent to participate}

Not applicable

\section{References}

[1] M Balch, D Tandy, A pose and position measurement system for the Hubble Space Telescope servicing mission, The International Society for Optical Engineering, 2007, vol. 6555

[2] J M. Kelsey, J Byrne, M Cosgrove, S Seereeram, R K. Mehra, VisionBased Relative Pose Estimation for Autonomous Rendezvous And Docking, IEEE Aerospace Conference Proceedings, 2006.

[3] G Arantes Jr., E M Rocco, I M. da Fonseca, S Theil. Far and proximity maneuvers of a constellation of service satellites and autonomous pose estimation of customer satellite using machine vision. Acta Astronautica, 2010, pp:1493-1505.

[4] Tongqun R, Jigui Z, Yingbiao G , et al. 3-D Free-form Shape Measuring System Using Unconstrained Range Sensor[J]. Chinese Journal of Mechanical Engineering, 2011(06):1095-1102.

[5] Zhao G , Xu S , Bo Y . LiDAR-Based Non-Cooperative Tumbling Spacecraft Pose Tracking by Fusing Depth Maps and Point Clouds[J]. Sensors, 2018, 18(10).

[6] Cao, Z, Yin, et al. Vision-based stabilization of nonholonomic mobile robots by integrating sliding-mode control and adaptive approach[J]. Chinese Journal of Mechanical Engineering, 2013.

[7] K Clemens, S Fredrik, D J Manuel, et al. SMART-OLEV-An orbital life extension vehicle for servicing commercial spacecrafts in GEO[J].
Acta Astronautica, 2007, 63(1-4):400-410.

[8] Sellmaier F., Boge T., Spurmann, J.,et al. On-orbit servicing missions: challenges and solutions for spacecraft operations[C]. SpaceOps 2010 Conference, Huntsville,AL, United states, 2010:1-11.

[9] Galante Joseph M, Van Eepoel J., Strube Matt, et al. Pose measurement performance of the argon relative navigation sensor suite in simulated flightconditions[R]. American Institute of Aeronautics and Astronautics, 2012. http://ntrs.nasa.gov/archive/nasa/casi.ntrs.nasa.gov/20120013578.pdf.

[10] Naasz Bo J., Van Eepoel J., Queen Steven Z., et al. Flight results from the HST SM4 relative navigation sensor system[C]. 33rd Annual AAS Rocky Mountain Guidance and Control Conference, Breckenridge,CO, 2010, 137:723-744.

[11] Chien Chiun-Hong. Target acquisition using natural feature image recognition[C]. Proceedings of IEEE Aerospace Conference, Big Sky, MT, United states, 2009:2707-2713.

[12] Y Jeong, D Lee, K Kim, et al. A wearable robotics arm with high force-reflection capability. Proceedings of the IEEE International Workshop on Robot and Human Interactive Communication, Osaka, Japan, September 27-29, 2000: 27-29.

[13] V Lepetit, F Moreno-Noguer, P Fua. EPnP: An Accurate O(n) Solution to the PnP Problem. International Journal of Computer Vision. 2009, 81, pp:155-166.

[14] Peng Chen, Guang-Da Hu, Jiarui Cui. Extended gravitational pose estimation. Optik, 2014,125, pp: 6106-6112.

[15] Yihong $\mathrm{Wu}$, Zhanyi ,Hu. PnP Problem Revisited, Journal of Mathematical Imaging and Vision, 2006, 24, pp:131-141.

[16] Long Quan and Zhongdan Lan. Linear N-Point Camera Pose Determination, IEEE Transactions on pattern analysis and machine intelligence, 1999, 21, pp:774-780.

[17] Ricardo Galego, Agustin Ortega, Ricardo Ferreira, Alexandre Bernardino, Juan Andrade-Cetto, José Gaspar. Uncertainty analysis of the DLT-Lines calibration algorithm for cameras with radial distortion, Computer Vision and Image Understanding, 2015, 140, pp:115-126.

[18] Juyang Weng, Narendra Ahuja, Thomas S. Huang. Optimal Motion and Structure Estimation, IEEE Transactions on pattern analysis and machine intelligence, 1993, 15, pp:864-884.

[19] Baojie Fan, Yingkui Du, Yang Cong. Robust and accurate online pose estimation algorithm via efficient three-dimensional collinearity model, IET Computer Vision, 2013, 7, pp:382-393.

[20] Chien-Ping Lu, Gregory D. Hager, Eric Mjolsness. Fast and Globally Convergent Pose Estimation from Video Images, IEEE Transactions on pattern analysis and machine intelligence, 2000, 22, pp:610-622.

[21] Zhang, Limin, Zhu, et al. Optimization-based non-cooperative spacecraft pose estimation using stereo cameras during proximity operations[J]. Applied Optics, 2017.

[22] Zhao G , Xu S , Bo Y . LiDAR-Based Non-Cooperative Tumbling Spacecraft Pose Tracking by Fusing Depth Maps and Point Clouds[J]. Sensors, 2018, 18(10).

[23] Capuano V , Alimo S R , Ho A Q , et al. Robust Feature Extraction for On-board Monocular-based Spacecraft Pose Acquisition[C]// AIAA Scitech 2019 Forum. 2019.

[24] Fadi Dornaika, Christophe Garcia. Pose Estimation using Point and Line Correspondences, Real-Time Imaging, 1999, 5, pp:215-230.

[25] Ilan Shimshoni, Ronen Basri, Member, Ehud Rivlin. A Geometric Interpretation of Weak-Perspective Motion, IEEE Transactions on pattern analysis and machine intelligence, 1999, 21, pp:252-257. 


\section{Biographical notes}

Li-Dong Mo, is currently a $\mathrm{PhD}$ candidate at School of mechanical and electrical engineering, Harbin Institute of Technology, Heilongjiang, China.

Tel: +86-182-22231667; E-mail: molidong@126.com

Guan-Xin Chi, is currently a professor at School of mechanical and electrical engineering, Harbin Institute of Technology, Heilongjiang, China. He received his $\mathrm{PhD}$ degree in Harbin Institute of Technology, China, in 1999.

Tel: +86-139-36130623; E-mail: chigx@hit.edu.cn

Zhen-Qing Zhao, is currently a teacher at Northeast Agricultural University, Heilongjiang, China.

Tel:+86-186-46031686; E-mail: zzq725@126.com 


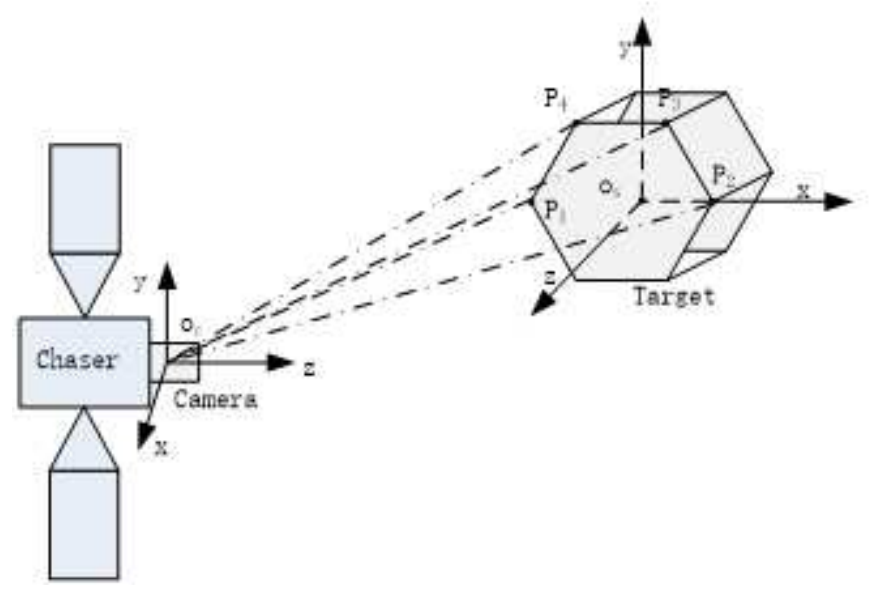

Figure 1

Target spacecraft and camera coordinate system

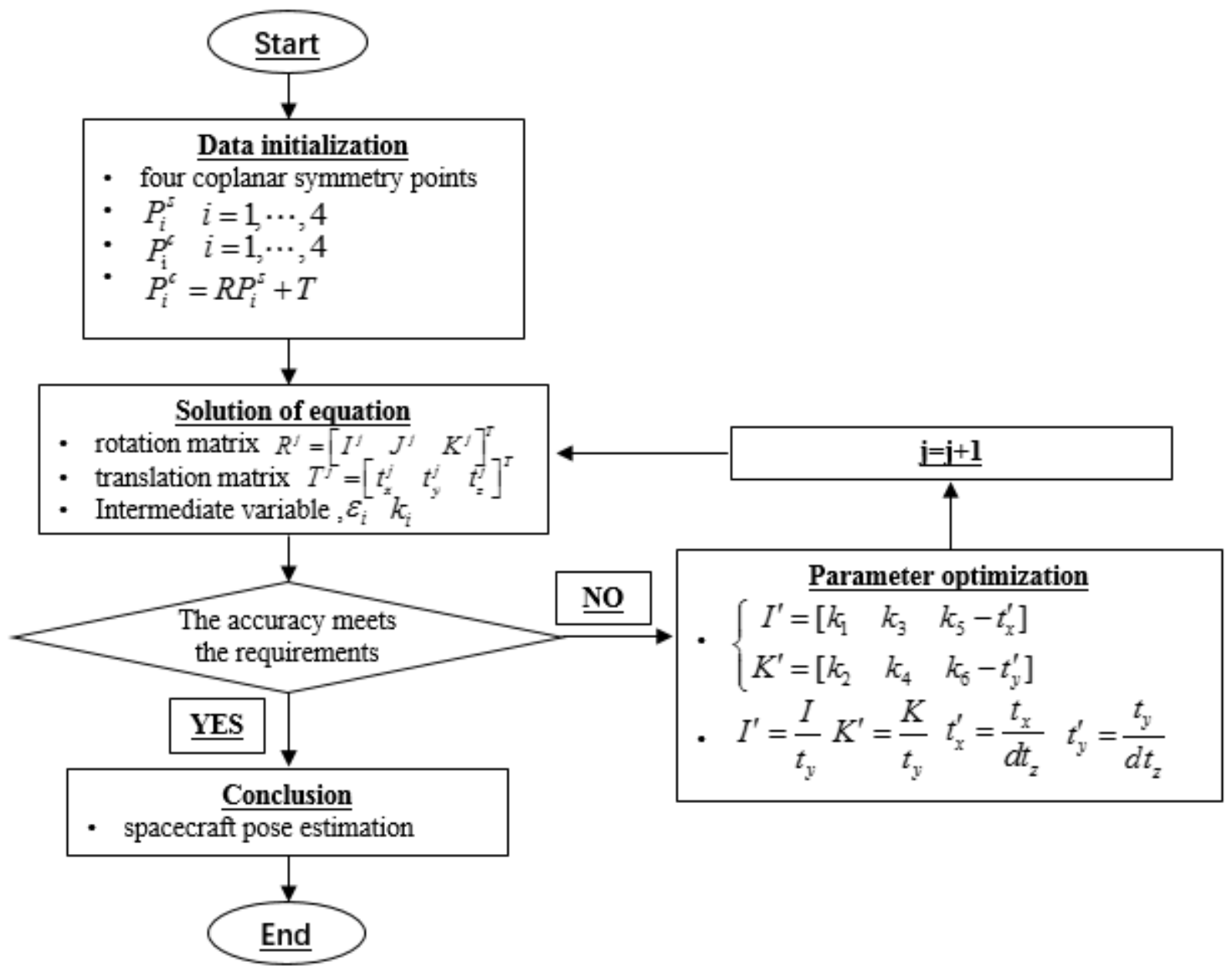

Figure 2

The optimal algorithm to improve the spacecraft pose estimation accuracy 


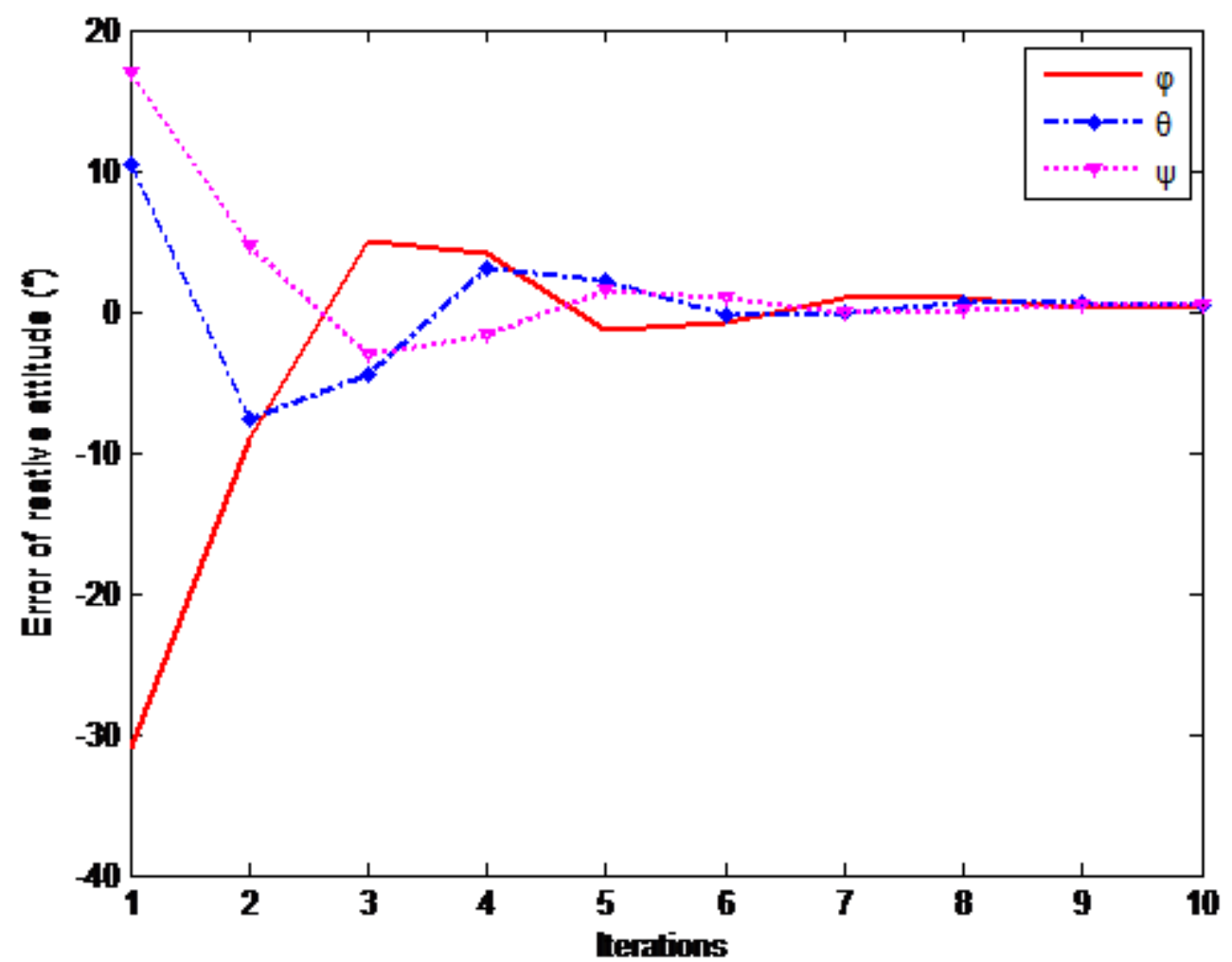

Figure 3

Attitude accuracy analysis using optimization.

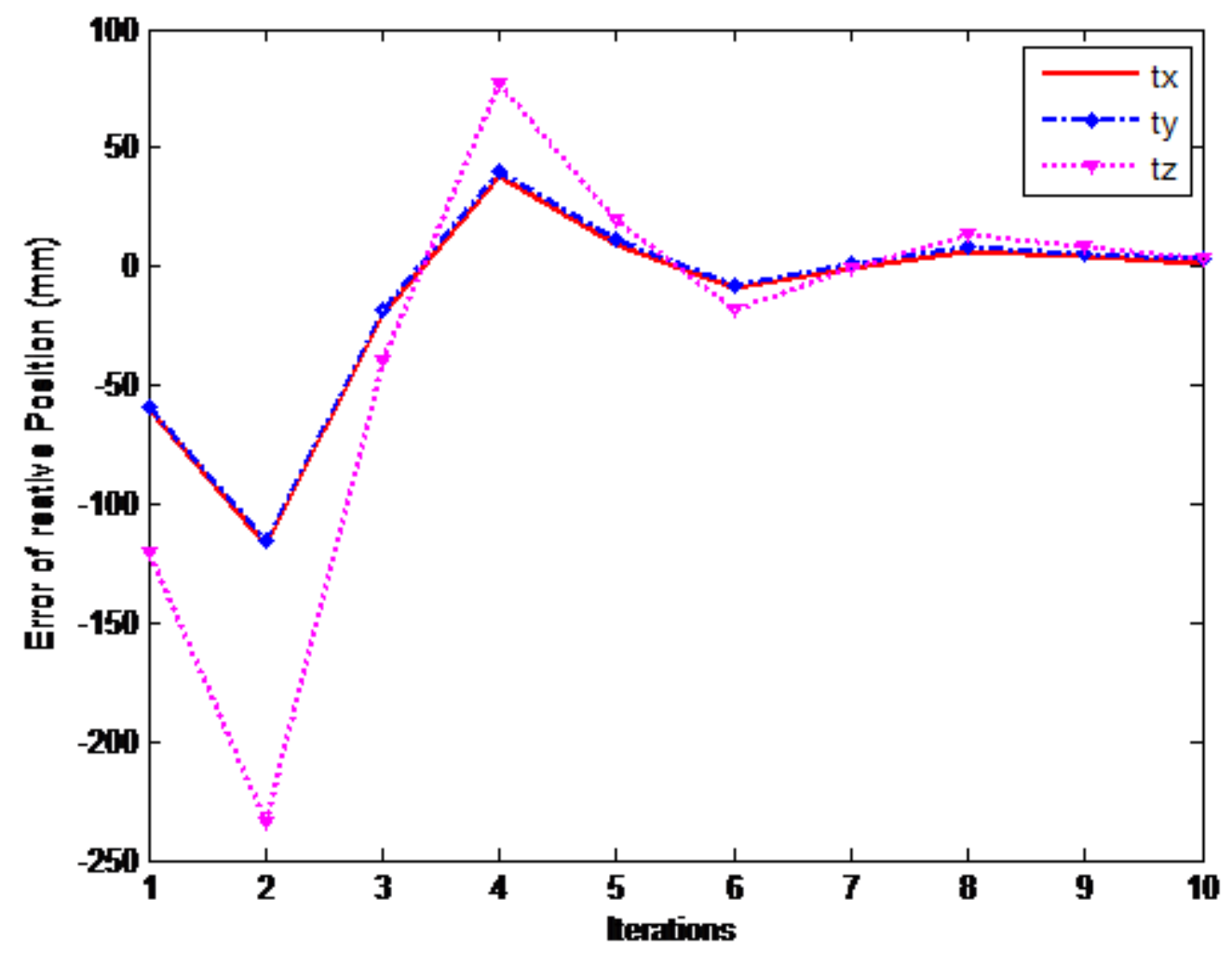


Figure 4

Position accuracy analysis using optimization.

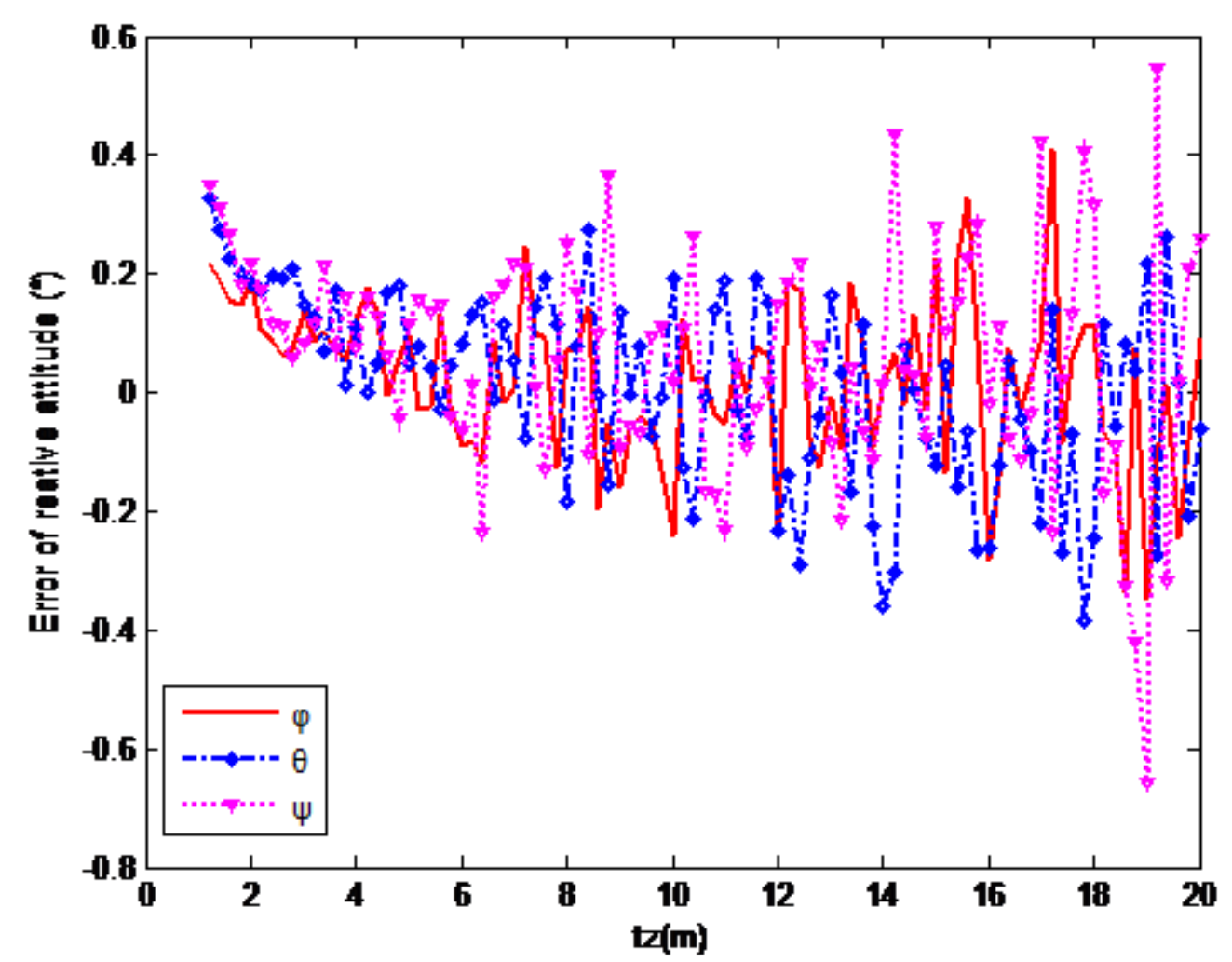

Figure 5

Attitude accuracy analysis with 0 mean and 0.1 pixel standard deviation noise 


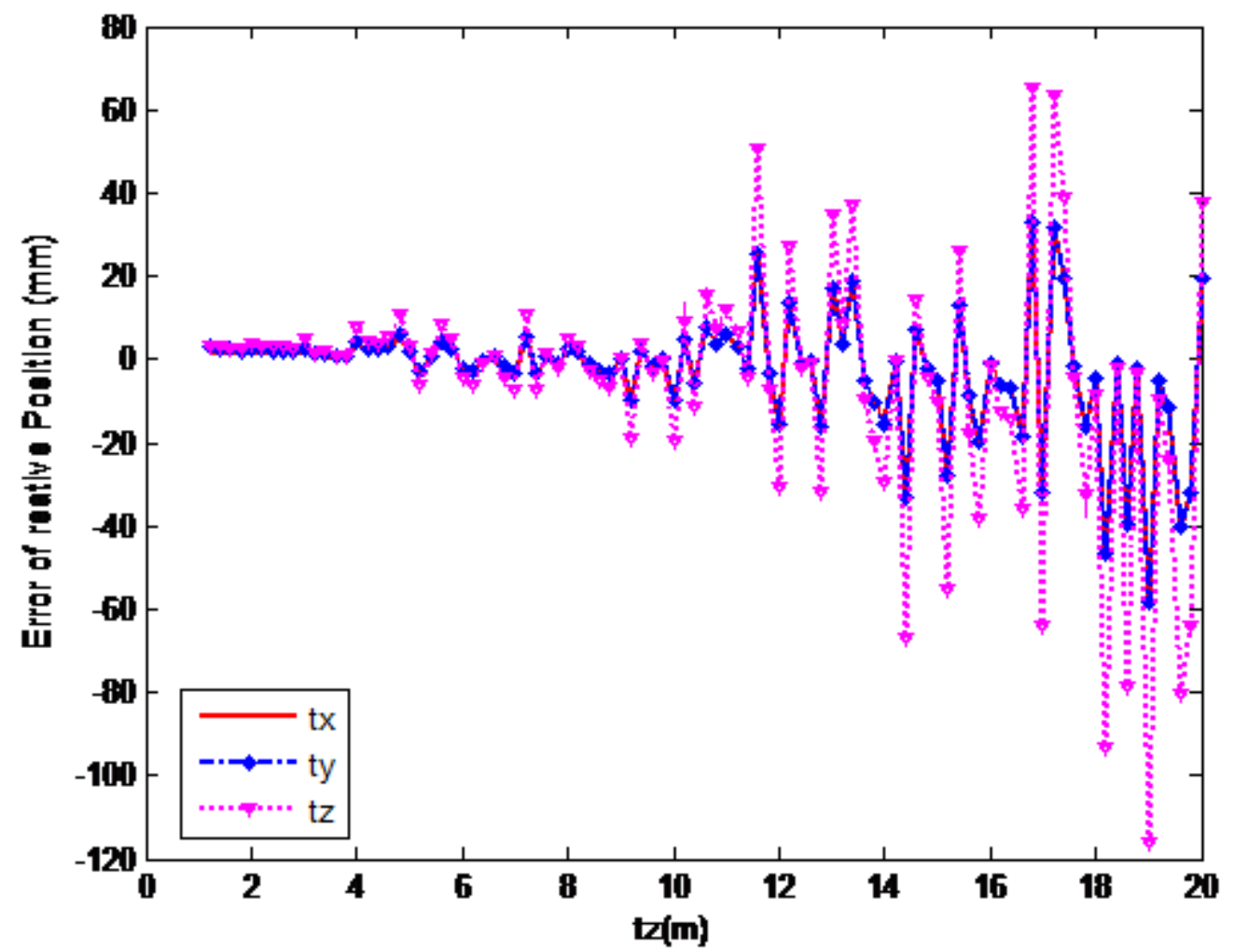

Figure 6

Position accuracy analysis with 0 mean and 0.1 pixel standard deviation noise

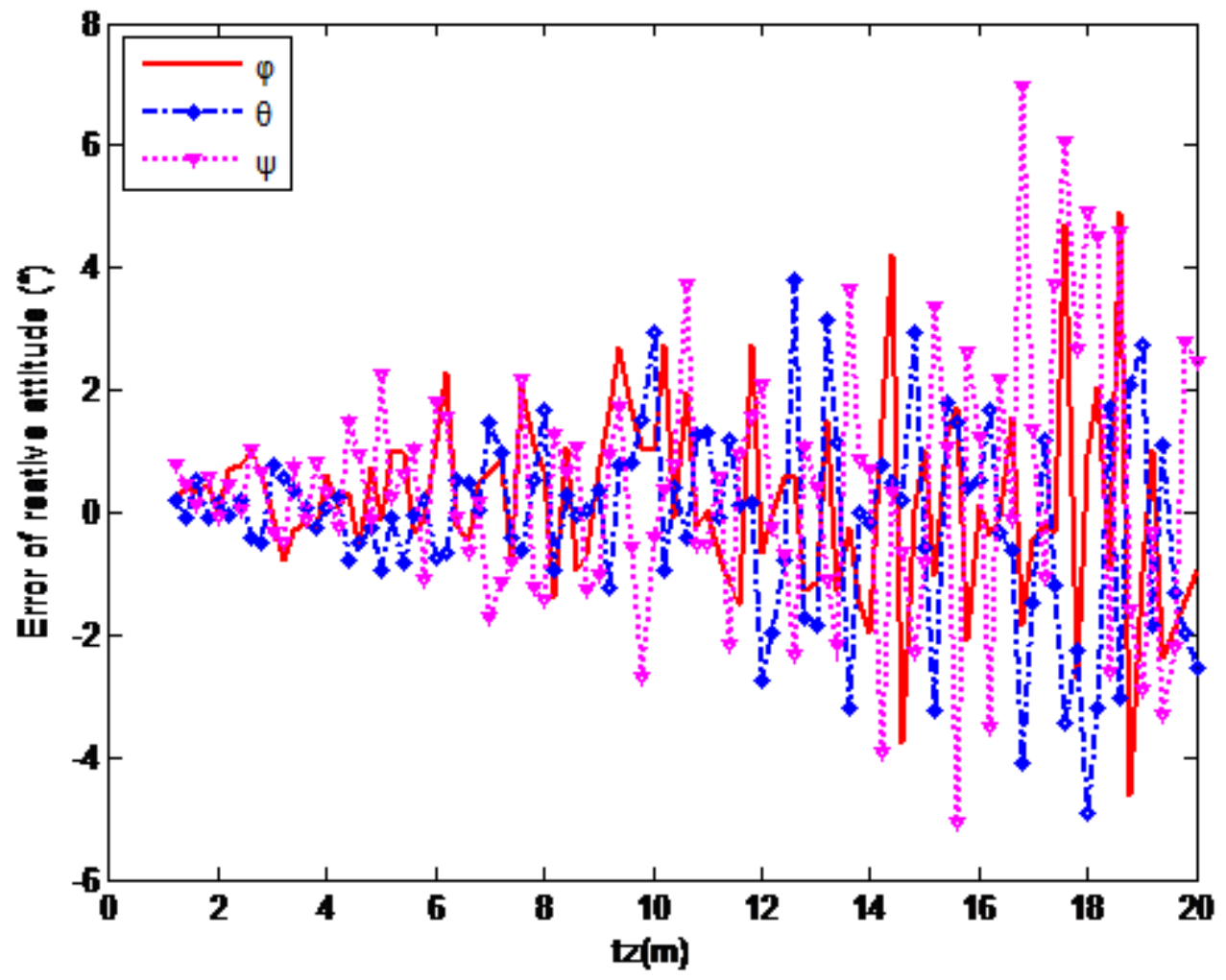


Figure 7

Attitude accuracy analysis with 0 mean and 1 pixel standard deviation noise

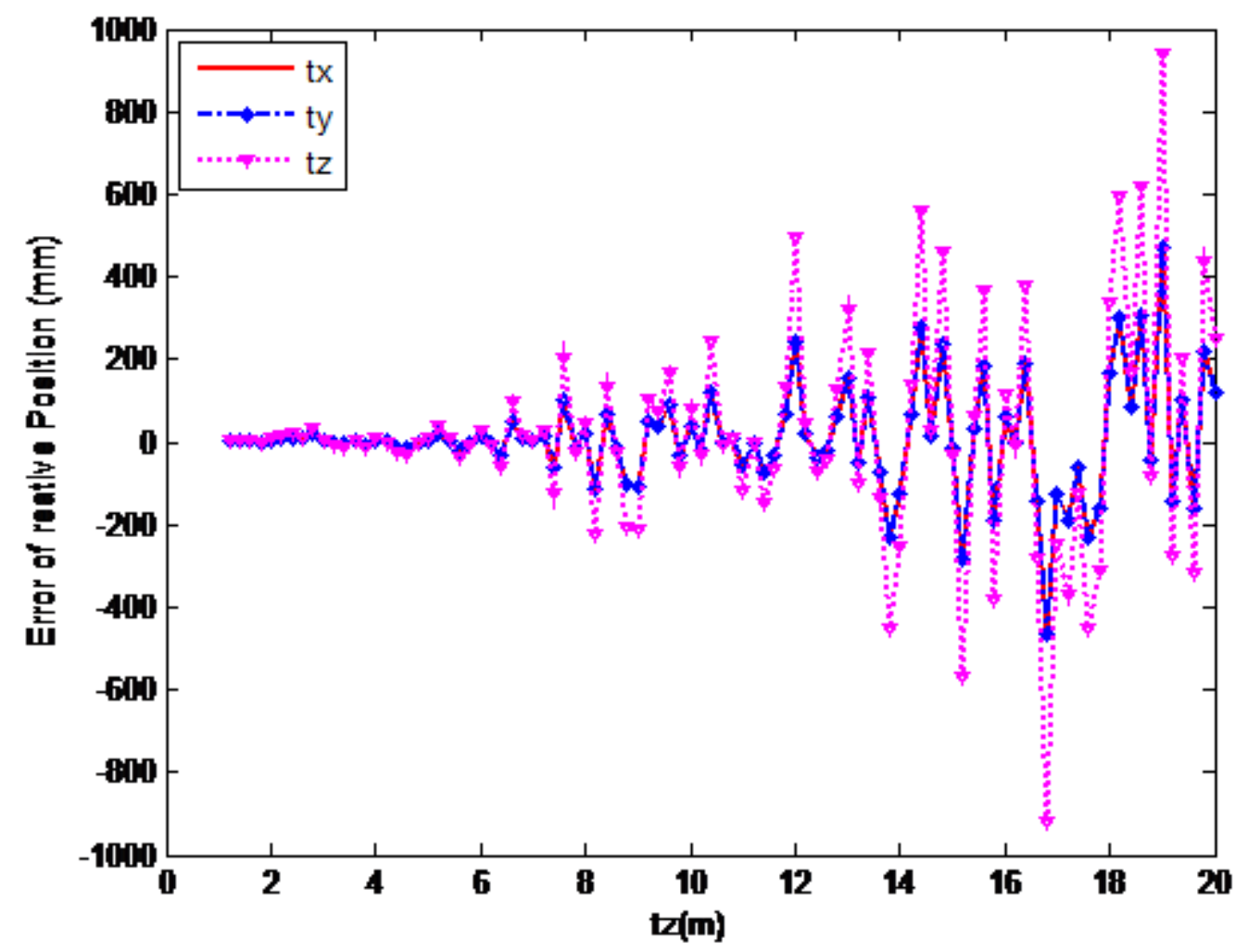

Figure 8

Position accuracy analysis with 0 mean and 1 pixel standard deviation noise 